\title{
A GENERALIZATION OF THE LEVINE-TRISTRAM LINK INVARIANT
}

\author{
LAWRENCE SMOLINSKY
}

\begin{abstract}
Invariants to $m$-component links are defined and are shown to be link cobordism invariants under certain conditions. Examples are given.
\end{abstract}

In knot and link theory there are certain signature invariants which determine up to torsion a knots cobordism class. These invariants were defined by Tristram [11], Levine [7] and Milnor [8] and are known as the Levine-Tristram or $p$-signatures. This paper investigates a generalization of the Levine-Tristram signature. The invariant can be viewed as a function which assigns $m$-roots of unity, one for each link component, to an integer. This invariant is a cobordism invariant in the same sense the Levine-Tristram signature is. However, unlike the Levine-Tristram signature, it is not a weak cobordism invariant. Our approach is geometric along the lines of O. Ja. Viro's geometric interpretation of the Levine-Tristram signature [12]. We discuss the relation of this invariant to the invariant of boundary links defined by Cappell and Shaneson in [2]. Applications of this invariant to the computation of Casson-Gordon invariants has been given in [9 and 10]. The author is grateful to J. Levine for many helpful discussions and to the referee for pointing out two errors.

Definitions. An $m$ component link of dimension $n$ or an $m$-link is an ordered collection of $m$ disjoint smooth oriented submanifolds of $S^{n+2}$, each of which is homeomorphic to $S^{n}$. We assume $n>2$ and our links will always be ordered. We will denote a link by $\left(S^{n+2} ; L_{1}, \ldots, L_{m}\right)$ when we wish to emphasize this point. We also write $L=L_{1} \cup L_{2} \cup \cdots \cup L_{m}$ to denote the link.

Every link bounds an oriented manifold called a Seifert surface. If an $m$ link has an $m$ component Seifert surface so that each component is bounded by exactly one link component then the link is a boundary link.

A link is sliced if it bounds a disjoint union of disks in the $(n+3)$-ball. A link is weakly sliced if it bounds a disk with punctures. An analysis of the obstructions for a link to be weakly sliced can be carried out for high dimensional links following the usual analysis for knots.

Received by the editors July 27, 1987 and, in revised form, March 14, 1988.

1980 Mathematics Subject Classification (1985 Revision). Primary 57Q45; Secondary 57M25, $57 \mathrm{Q} 60$.

Key words and phrases. Link invariants, link cobordism, boundary link cobordism. 


\section{THE LINK INVARIANT}

Let $\mathscr{S}$ be either the sphere $S^{n+2}$ or the disk $D^{n+2}$ and suppose $V_{1}, \ldots, V_{m}$ are codimension two submanifolds of $\mathscr{S}$. We require the $V_{i}$ to satisfy:

(1) $V_{i} \cap V_{j}=\varnothing$ for $i \neq j$;

(2) $\nu\left(V_{i}\right) \subset \mathscr{S}$ is trivial;

(3) $V_{i} \cap \partial V_{i}=\partial V_{i}$.

We denote such an ordered set of submanifolds $\left(\mathscr{S} ; V_{1}, \ldots, V_{m}\right)$ and call this collection a special $m$-tuple. $H_{1}\left(\mathscr{S}-\left(V_{1} \cup \cdots \cup V_{m}\right)\right)=Z^{m}$ by Alexander duality, so let $\mu_{i}$ be the class of the meridian to $V_{i}$. Note that an $m$-link $\left(S^{n+2} ; L_{1}, \ldots, L_{m}\right)$ is a special $m$-tuple and here $\mu_{i}$ is the meridian of $L_{i}$.

We will discuss finite abelian groups $G=Z_{a_{1}} \oplus \cdots \oplus Z_{a_{m}}$ but we really wish to consider $m$-tuples of cyclic groups $\left(Z_{a_{1}}, \ldots, Z_{a_{m}}\right)$. The order is important and we indicate it by the ordering of the summands of $G$. We also choose preferred generators for $G$; let $g_{i}$ be the generator of $Z_{a_{i}}$.

Proposition 1. Let $\left(\mathscr{S} ; V_{1}, \ldots, V_{m}\right)$ be a special m-tuple and let $G=Z_{a_{1}} \oplus$ $\cdots \oplus Z_{a_{m}}$ be a finite abelian group with one summand of $G$ corresponding to one submanifold of $\mathscr{S}$. Then there is a canonical $G$-manifold $M$ that has the following properties:

(1) $M \stackrel{\pi}{\rightarrow} M / G=\mathscr{S}$.

(2) $M-\pi^{-1}\left(\bigcup_{i} V_{i}\right) \rightarrow \mathscr{S}-\left(\bigcup_{i} V_{i}\right)$ is a regular covering space with group $G$.

(3) $\pi^{-1}\left(V_{i}\right) \stackrel{\pi}{\rightarrow} V_{i}$ is a regular covering space with group $G / Z_{a_{i}}$.

Remark. Proposition 1 only requires $\mathscr{S}$ to be an $(n+2)$-manifold with $H^{1}(\mathscr{S})$ $=0$ and the $V_{i}$ to satisfy (1), (2) and (3).

Proof. Let $\lambda_{j}: H_{1}\left(\mathscr{S}-\bigcup V_{i}\right) \rightarrow Z$ take the linking coefficient of a class in $H_{1}\left(\mathscr{S}-\cup V_{i}\right)$ with the fundamental class of $V_{j}$. The map $\left(\lambda_{1}, \ldots, \lambda_{m}\right)$ : $H_{1}\left(\mathscr{S}-\cup V_{i}\right) \rightarrow Z^{m}$ is an isomorphism by Alexander duality. Let $\widehat{M}$ be the covering space which corresponds to the kernel of the composition,

$$
\pi_{1}\left(\mathscr{S}-\bigcup V_{i}\right) \stackrel{h}{\rightarrow} H_{1}\left(\mathscr{S}-\bigcup V_{i}\right) \stackrel{\left(\lambda_{1}, \ldots, \lambda_{m}\right)}{\longrightarrow} Z \stackrel{m}{\longrightarrow} \stackrel{\bmod }{\longrightarrow} G .
$$

In this composition $h$ is the Hurewicz homomorphism and mod is the reduction of the $i$ th coordinate modulo $a_{i}$. The covering translations are $G$ and have canonical generators arising from the meridians $\mu_{i}$.

Framings of $\nu\left(V_{j}\right)$ are given by elements of $\left[V_{j}, S^{1}\right]$. If $f \in\left[V_{j}, S^{1}\right]$ is a framing define $p_{f}: V_{j} \rightarrow \nu\left(V_{j}\right)-V_{j}$ by pushing $V_{j}$ according to the framing $f$. Now,

$$
\begin{aligned}
{\left[V_{j}, S^{1}\right] } & \stackrel{\sim}{\rightarrow} \operatorname{Hom}\left(H_{1}\left(V_{j}\right), H_{1}\left(S^{1}\right)\right) \\
f & \longmapsto f_{*}
\end{aligned}
$$


and $\operatorname{Hom}\left(H_{1}\left(V_{j}\right), H_{1}\left(S^{1}\right)\right)=\operatorname{Hom}\left(H_{1}\left(V_{j}\right), Z\right)$ since $S^{1}$ is a meridian and is so oriented. $\lambda_{j}\left(\left[S^{1}\right],\left[V_{j}\right]\right)=1$ so $f_{*}(a)=\lambda_{j}\left(p_{f *}(a),\left[V_{j}\right]\right)$. We choose the framing $f$ so that $\lambda_{j}\left(-,\left[V_{j}\right]\right) \circ p_{f *}: H_{1}\left(V_{j}\right) \rightarrow Z$ is zero.

We now examine the induced cover on $V_{j} \times\left(B^{2}-0\right)$, where 0 is the origin in $B^{2}$. The composition,

$$
\bmod \circ\left(\lambda_{1}, \ldots, \lambda_{m}\right): H_{1}\left(V_{j} \times\left(B^{2}-0\right)\right) \rightarrow G
$$

generates the covering. $H_{1}\left(V_{j} \times\left(B^{2}-0\right)\right) \approx H_{1}\left(V_{j}\right) \oplus H_{1}\left(B^{2}-0\right)$ and the induced maps on the summands are given by $\lambda_{j}: H_{1}\left(B^{2}-0\right) \rightarrow Z_{a_{j}} \subset G$ and the composition

$$
H_{1}\left(V_{j}\right) \stackrel{\left(\lambda_{1}, \ldots, \lambda_{j-1}, \lambda_{j+1}, \ldots, \lambda_{m}\right)}{\longrightarrow} Z^{m-1} \rightarrow Z_{a_{1}} \oplus \cdots \oplus Z_{a_{j-1}} \oplus Z_{a_{j+1}} \oplus \cdots \oplus Z_{a_{m}} .
$$

The last group, the image of the composition, we denote $H_{j}$. The generator of $H_{1}\left(B^{2}-0\right)$ is a meridian of $V_{j}$, which does not link with any other $V_{i}$, i.e., $\lambda_{i}: H_{1}\left(B^{2} \times 0\right) \rightarrow Z_{a_{i}}$ is zero. In the second map, $H_{1}\left(V_{j}\right) \rightarrow Z_{a_{j}}$ is zero by the choice of framing. The induced (possibly disconnected) cover of $V_{j} \times\left(B^{2}-0\right)$ is therefore a product of the $H_{j}$-cover of $V_{j}$ and the $Z_{a_{j}}$-cover of $B^{2}-0$.

We can now extend the cover of $V_{j} \times\left(B^{2}-0\right)$ to a $G$-manifold by extending the $Z_{a_{j}}$-cover of $B^{2}-0$ to the standard $Z_{a_{j}}$ branched cover of $\left(B^{2}, 0\right)$.

$$
\begin{gathered}
\left(Z_{a_{j}} \text {-cover of } B^{2}-0\right) \times\left(H_{j} \text {-cover of } V_{j}\right) \rightarrow\left(B^{2}-0\right) \times V_{j} \\
\Pi \\
\left(Z_{a_{j}} \text {-branched cover of } B^{2}\right) \times\left(H_{j} \text {-cover of } V_{j}\right) \rightarrow B^{2} \times V_{j}
\end{gathered}
$$

Repeating the process of completing the covers of $\nu\left(V_{i}\right)-V_{i}$ for all $i$ yields the $G$-manifold $M$. From the construction it is easy to see that $M$ satisfies conditions (1), (2), and (3).

Remark. $\pi^{-1}\left(V_{j}\right) \rightarrow V_{j}$ is the covering space with group $H_{j}$ gotten from the linking numbers with the other components. In particular, if these are zero then $\pi^{-1}\left(V_{j}\right)=V_{j} \times H_{j}$ is a trivial cover.

The following lemma is well known.

Lemma 2. Let $\left(S^{n+2} ; L_{1}, \ldots, L_{m}\right)$ be a link with $n>1$ or $\lambda\left(L_{i}, L_{j}\right)=0$ for all $i$ and $j$. There exist $m$ disjoint submanifolds in $B^{n+3}$ so that $\left(B^{n+3} ; V_{1}, \ldots\right.$, $\left.V_{m}\right)$ is a special $m$-tuple and $\left(\partial B^{n+3} ; \partial V_{1}, \ldots, \partial V_{m}\right)=\left(S^{n+3} ; L_{1}, \ldots, L_{m}\right)$.

We can now associate $G$-manifolds to links. Let $G=Z_{a_{1}} \oplus \cdots \oplus Z_{a_{m}}$ so there is one summand to correspond to each component of a link $L=L_{1} \cup \cdots \cup$ $L_{m} \subset S^{n+2}$. Let $\left(B^{n+3} ; V_{1}, \ldots, V_{m}\right)$ be a special $m$-tuple and $\partial\left(B^{n+3}, V_{i}\right)=$ $\left(S^{n+2}, L_{i}\right)$. The canonical $G$-manifold over $\left(B^{n+3} ; V_{1}, \ldots, V_{m}\right)$ is a $G$-manifold associated to $L$. 
Denote by [, ] the inner product of characters for a group $G$. If $M$ is a $2 k$ dimensional $G$-manifold we write $\operatorname{Sign}(G, M)$ for the $G$-signature of $M$, see [1]. Let $\chi$ be an irreducible character and $V_{\chi}=\left\{v \in H_{k}(M ; \mathbf{R}) \otimes \mathbf{C}: g_{*}(v)=\right.$ $\chi(g)(r)$ for all $g \in G\}$. Then

$$
[\operatorname{Sign}(G, M), \chi]=\operatorname{signature}\left(\left.\langle,\rangle\right|_{V_{\chi}}\right)
$$

where $\langle$,$\rangle is the intersection pairing if k$ is even or $i$ times the intersection pairing if $k$ is odd. Note that

$$
V_{\chi}=\bigcap_{i=1}^{m}\left(w_{i} \text { eigenspace of } g_{i}\right)
$$

where $w_{i}=\chi\left(g_{i}\right)$ is an $a_{i}$ th root of unity.

Definition. Suppose $L$ is a $2 q-1$ dimensional $m$-link and $G=Z_{a_{1}} \oplus \cdots \oplus Z_{a_{m}}$ has $g_{i}$ as the generator of $Z_{a_{i}}$. Let $W$ be a $G$-manifold associated to the link $L$ and $\chi$ an irreducible character of $G$. We define

$$
\operatorname{sig}(L, \chi)=[\operatorname{Sign}(G, W), \chi] .
$$

This invariant is well defined by

Theorem 3. If $M_{V}$ and $M_{V^{\prime}}$ are G-manifolds associated to a link then $\operatorname{Sign}\left(G, M_{V}\right)=\operatorname{Sign}\left(G, M_{V^{\prime}}\right)$. Hence the link invariant is independent of the choice of manifolds which span the link.

The proof of this theorem relies on a lemma and the additivity of the $G$ signature.

Additivity Property (Proposition 7.1 in [1]). If $X, Y$ and $Z$ are $G$-manifolds with $\partial X=\partial Y=Z$ then

$$
\operatorname{Sign}\left(G, X \cup_{\mathrm{z}}(-Y)\right)=\operatorname{Sign}(G, X)-\operatorname{Sign}(G, Y) .
$$

Lemma 4. If $\left(S^{2 q+2} ; W_{1}, \ldots, W_{m}\right)$ is a special $m$-tuple and $M_{W}$ is its canonical $G$-manifold then $\operatorname{Sign}\left(G, M_{W}\right)=0$.

Proof of Theorem 3. If $M_{V}$ and $M_{V^{\prime}}$ are constructed from $\left(D^{2 q+2} ; V_{1}, \ldots, V_{m}\right)$ and $\left(D^{2 q+2} ; V_{1}^{\prime}, \ldots, V_{m}^{\prime}\right)$ respectively then $M_{V} \cup\left(-M_{V^{\prime}}\right)$ is the $G$-manifold over $\left(S^{2 q+2} ; V_{1} \cup\left(-V_{1}^{\prime}\right), \ldots, V_{m} \cup\left(-V_{M}^{\prime}\right)\right)$ where $M_{L}$ is the $G$-manifold over $\left(S^{2 q+1} ; L_{1}, \ldots, L_{m}\right)=\partial\left(D^{2 q+2} ; V_{1}, \ldots, V_{m}\right)=\partial\left(D^{2 q+2} ; V_{1}^{\prime}, \ldots, V_{m}^{\prime}\right)$. Note that $\nu\left(V_{i} \cup\left(-V_{i}^{\prime}\right)\right) \subset S^{2 q+2}$ is trivial since the bundle is formed by glueing $\nu\left(S^{2 q-1}\right) \subset \nu\left(V_{i}\right)$ to $\nu\left(S^{2 q-1}\right) \subset \nu\left(V_{i}^{\prime}\right)$ and this glueing is specified by an element of $\pi_{2 q-1}(0(2))$ which is 0 . The lemma and the additivity property imply $\operatorname{Sign}\left(G, M_{V}\right)=\operatorname{Sign}\left(G, M_{V^{\prime}}\right)$.

Proof of Lemma 4. We consider $\left(S^{2 q+2} ; W_{1}, \ldots, W_{m}\right)$ as arising via the ThomPontryagin construction from regular values $p_{i} \in S_{i}^{2}$ and a map $f: S^{2 q+2} \rightarrow$ 
$\bigvee_{i=1}^{m} S_{i}^{2}$. One can then define a map,

$$
F: \pi_{2 q+2}\left(\bigvee_{i=1}^{m} S^{2}\right) \rightarrow R(G)
$$

Suppose $\gamma \in \pi_{2 q+2}\left(\bigvee_{i=1}^{m} S_{i}^{2}\right)$ and choose a $g: S^{2 q+2} \rightarrow \bigvee S^{2}$ so $[g]=\gamma$ and $g$ has $p_{i} \in S_{i}^{2}$ as regular values. We then get a special $m$-tuple $\left(S^{2 q+2} ; g^{-1}\left(p_{1}\right)\right.$, $\left.\ldots, g^{-1}\left(p_{m}\right)\right)$ and $M_{g}$ the canonical $G$-manifold over this special $m$-tuple. Then we define $F$ by

$$
F(\gamma)=\operatorname{sign}\left(G, M_{g}\right) \text {. }
$$

If $g_{0}$ and $g_{1}$ both represent $\gamma$ then there is a homotopy $H: S^{2 q+2} \times I \rightarrow \bigvee S^{2}$ so that the $p_{i} \in S_{i}^{2}$ are regular values, $H_{0}=g_{0}$ and $H_{1}=g_{1}$. Let $M_{H}$ be the canonical $G$-manifold over $\left(S^{2 q+2} \times I ; H^{-1}\left(p_{1}\right), \ldots, H^{-1}\left(p_{m}\right)\right)$. Since $\partial M_{H}=M_{g_{0}} \cup\left(-M_{g_{1}}\right)$ we have by additivity, $\operatorname{Sign}\left(G, \partial M_{H}\right)=\operatorname{Sign}\left(G, M_{g_{0}}\right)-$ $\operatorname{Sign}\left(G, M_{g_{1}}\right) . \partial M_{H}$ is the boundary of a $G$-manifold and so has zero $G$ signature. Therefore $F$ is a well-defined function.

$F$ is a homomorphism. If $\gamma_{1}$ is represented by the map $g_{1}$ with corresponding $m$-tuple $\left(S^{2 q+2} ; P_{1}, \ldots, P_{m}\right)$ and $\gamma_{2}$ is represented by the map $g_{2}$ with corresponding $m$-tuple $\left(S^{2 q+2} ; Q_{1}, \ldots, Q_{m}\right)$ then $\gamma_{1}+\gamma_{2}$ is represented by a map $h$ with corresponding $m$-tuple $\left(S^{2 q+2} \# S^{2 q+2} ; P_{1} \cup Q_{1}, \ldots, P_{m} \cup Q_{m}\right)$. The connected sum is taken by removing disks disjoint from the $\nu\left(P_{i}\right)$ 's and $\nu\left(Q_{i}\right)$ 's. $M_{g_{1}} \stackrel{\pi}{\rightarrow} S^{2 q+2}$ is a covering space away from $\bigcup \nu\left(P_{i}\right)$ so $\pi^{-1}$ (removed disk) is a union of $|G|$ disks in $M_{g_{1}}$. The same holds true for $M_{g_{2}}$. Now $M_{h}=\left(M_{g_{1}}-\right.$ $|G|$ disks $) \cup\left(M_{g_{2}}-|G|\right.$ disks $)$ where the union is taken along the boundary of the disks. Since $\operatorname{Sign}(G,|G|$ disks $)=0, \operatorname{Sign}\left(G, M_{g_{i}}-\operatorname{disks}\right)=\operatorname{Sign}\left(G, M_{g_{i}}\right)$. By the additivity of the $G$-signature, $\operatorname{Sign}\left(G, M_{h}\right) \stackrel{g_{i}}{=} \operatorname{Sign}\left(G, M_{g_{1}}\right)+\operatorname{Sign}\left(G, M_{g_{2}}\right)$.

We now show that $F$ is the zero map. By the Hilton-Milnor theorem, $\pi_{2 q+2}\left(\bigvee S^{2}\right) \approx \bigoplus_{j}\left(\bigoplus \pi_{2 q+2}\left(S^{j+1}\right)\right)$ where the inner sum is over certain $(j-1)$ iterated Whitehead products [13]. Since $2 q+2$ is even, the only summands we must check are $\pi_{2 q+2}\left(S^{2 q+2}\right)$. They are the only infinite summands and $R(G)$ has no torsion. If $i_{k}: S^{2} \rightarrow S_{1}^{2} \vee \cdots \vee S_{k}^{2} \vee \cdots \vee S_{m}^{2}$ is the inclusion into the $k$ th spot then the maps, $\pi_{2 q+2}\left(S^{2 q+2}\right) \hookrightarrow \pi_{2 q+2}\left(\bigvee S^{2}\right)$, are given by $\beta \rightarrow w\left(i_{1}, \ldots, i_{m}\right) \circ \beta$ where $w\left(i_{1}, \ldots, i_{m}\right)$ is an iterated Whitehead product of the $i_{j}$,'s with one Whitehead product corresponding to one summand inclusion. Since it is only necessary to check the generators of $\pi_{2 q+2}\left(S^{2 q+2}\right)$, we reduced the problem is showing $F\left(w\left(i_{1}, \ldots, i_{m}\right)\right)=0$. We make the following claim which we prove shortly.

Claim. The manifolds $w\left(i_{1}, \ldots, i_{m}\right)^{-1}\left(p_{j}\right)$ are products of spheres.

Since all the manifolds contained in the special $m$-tuple $\left(S^{2 q+2} ; N_{1}, \ldots, N_{m}\right)$ associated to $w\left(i_{1}, \ldots, i_{m}\right)$ are products of spheres, so are the covering spaces 
of the $N_{j}$ 's. The various fixed point sets of the canonical $G$-manifold over $\left(S^{2 q+2} ; N_{1}, \ldots, N_{m}\right)$ are covers of the $N_{j}$ 's and so have no signature. In addition the normal bundles to the fixed point sets are trivial, so by the $G$-signature theorem [1], $\operatorname{Sign}\left(G, M_{N}\right)=0$.

We now prove the claim. Suppose $[f] \in \pi_{n+1}\left(\bigvee S^{2}\right)$ and $[g] \in \pi_{m+1}\left(\bigvee S^{2}\right)$ so that $f:\left(D^{n+1}, \partial D^{n+1}\right) \rightarrow\left(\bigvee S^{2}, *\right)$ and $g:\left(D^{m+1}, \partial D^{m+1}\right) \rightarrow\left(\bigvee S^{2}, *\right)$ have $p$ as a regular value. We also assume $f^{-1}(p)$ and $g^{-1}(p)$ are a union of products of spheres, or one or both may be a point.

We write $S^{n+m+1}$ as $D^{n+1} \times \partial D^{m+1} \bigcup_{\partial D^{n+1} \times \partial D^{m+1}} \partial D^{n+1} \times D^{m+1}$. On each section the Whitehead product $[f, g] \in \pi_{n+m+1}\left(\bigvee S^{n}\right)$ is

$$
\left(D^{n+1} \times \partial D^{m+1}, \partial D^{n+1} \times \partial D^{m+1}\right) \stackrel{\pi_{n}}{\rightarrow}\left(D^{n+1}, \partial D^{n+1}\right) \stackrel{f}{\rightarrow}(\bigvee S, *)
$$

and

$$
\left(\partial D^{n+1} \times D^{m+1}, \partial D^{n+1} \times \partial D^{m+1}\right) \stackrel{\pi_{n}}{\rightarrow}\left(D^{m+1}, \partial D^{m+1}\right) \stackrel{g}{\rightarrow}\left(\bigvee S^{2}, *\right) .
$$

So $[f, g]^{-1}(p)=\left(f \circ \pi_{n}\right)^{-1}(p) \cup\left(g \circ \pi_{m}\right)^{-1}(p)$. Now, $\left(f \circ \pi_{n}\right)^{-1}(p)=\pi_{n}^{-1}\left(f^{-1}(p)\right)$ $=\partial D^{m+1} \times f^{-1}(p)$ which is a union of products of spheres. The same is true of $\left(g \circ \pi_{m}\right)^{-1}(p)=\partial D^{n+1} \times g^{-1}(p)$.

\section{THE LEVINE-TRISTRAM SIGNATURE AND LINK COBORDISM}

Let $L$ be a $2 q-1$ dimensional $m$-link and $\Omega=\left(\omega_{1}, \ldots, \omega_{m}\right)$ an $m$-tuple with $\omega_{i}$ an $a_{i}$-root of unity. Suppose $G=Z_{a_{1}} \oplus \cdots \oplus Z_{a_{m}}$ with $g_{i}$ the preferred generator of $Z_{a_{i}}$ and $\chi_{G}\left(g_{i}\right)=\omega_{i}$.

Definition. $\operatorname{sig}_{L}(\Omega)=\operatorname{sig}\left(L, \chi_{G}\right)$.

We pause to introduce new notation. If $V$ is a vectorspace with a $G$ action we write $E_{\Omega}(V)$ for the eigenspace intersection $\bigcap_{i=1}^{n}\left(\omega_{i}\right.$ eigenspace of $\left.g_{i}\right)$. If $M$ is a $G$-manifold then we sometimes write $E_{\Omega}(M)$ for $E_{\Omega}\left(H_{*}(M ; \mathbf{C})\right)$ or $E_{\Omega}\left(H^{*}(M ; \mathbf{C})\right)$.

Lemma 5. Let $\Omega$ be an m-tuple of roots of unity with $\omega_{i}$ a primitive $a_{i}$ th root of unity. Let $\left(D^{2 q+2} ; V_{1}, \ldots, V_{m}\right)$ be a special $m$-tuple associated to a link $L^{2 q-1}$ and $\phi\left(H_{1}\left(D^{2 q+2}-\left(V_{1} \cup \cdots \cup V_{m}\right)\right)\right) \rightarrow G$ by $\phi\left(\mu_{i}\right)=g_{i}$. If $\widetilde{X} \rightarrow D-\left(\bigcup_{i=1}^{m} V_{i}\right)$ is the covering space induced by $\phi$ then

$$
\operatorname{sig}_{L}(\Omega)=\operatorname{sign}\left(\left.\langle,\rangle\right|_{E_{\Omega}\left(H_{q+1}(\widetilde{X}, \mathbf{C})\right)}\right) .
$$

Proof. Note that if $\omega_{i}=1$ for all $i$ then $\operatorname{sig}_{L}(\Omega)=0$ and $\tilde{X} \subset D^{2 q+2}$ has zero intersection matrix. The formula follows in this case.

Let $M$ be the canonical $G$-manifold over our special $m$-tuple and consider the Mayer-Vietoris sequence arising from

$$
0 \rightarrow \Delta\left(\rho^{-1} \partial\left(\nu\left(\bigcup_{i} V_{i}\right)\right)\right) \rightarrow \Delta\left(\rho^{-1}\left(\nu\left(\bigcup_{i} V_{i}\right)\right)\right) \oplus \Delta(\tilde{X}) \rightarrow \Delta(M) \rightarrow 0 .
$$


Now, $\rho^{-1}\left(\nu\left(\bigcup_{i} V_{i}\right)\right)=\bigcup_{i} D^{2} \times \widetilde{V}_{i}$ and $\rho^{-1}\left(\partial \nu\left(\bigcup_{i} V_{i}\right)\right)=\bigcup_{i} S^{1} \times \widetilde{V}_{i}$. We get the following equivariant exact sequence:

$$
\begin{aligned}
\bigoplus_{i} H_{q+1}\left(S^{1} \times \widetilde{V}_{i}\right) & \rightarrow \bigoplus_{i} H_{q+1}\left(D^{2} \times \widetilde{V}_{i}\right) \oplus H_{q+1}(\tilde{X}) \\
& \rightarrow H_{q+1}(M) \rightarrow \bigoplus_{i} H_{q}\left(S^{1} \times \widetilde{V}_{i}\right) \rightarrow
\end{aligned}
$$

and the following exact sequence of eigenspace intersections,

$$
\begin{aligned}
\bigoplus_{i} E_{\Omega} H_{q+1}\left(S^{1} \times \widetilde{V}_{i}\right) & \rightarrow \bigoplus_{i} E_{\Omega} H_{q+1}\left(D^{2} \times \widetilde{V}_{i}\right) \oplus E_{\Omega} H_{q+1}(\tilde{X}) \\
& \rightarrow E_{\Omega} H_{q+1}(M) \rightarrow \bigoplus_{i} E_{\Omega} H_{q}\left(S^{2} \times \widetilde{V}_{i}\right) .
\end{aligned}
$$

Now, $g_{i}: D^{2} \times \widetilde{V}_{i} \rightarrow D^{2} \times \widetilde{V}_{i}$ is rotation in $D^{2}$ and $g_{i}: S^{1} \times \widetilde{V}_{i} \rightarrow S^{1} \times \widetilde{V}_{i}$ is rotation in $S^{1}$ so if $v \in E_{\Omega} H_{*}\left(D^{2} \times \widetilde{V}_{i}\right)$ or $E_{\Omega} H_{*}\left(S^{1} \times \widetilde{V}_{i}\right)$ then $g_{i *}(v)=v$ but $g_{i *}(v)=\omega_{i} v$. Since $\omega_{i} \neq 1$ for all $i, E_{\Omega} H_{*}\left(D^{2} \times \widetilde{V}_{i}\right)$ and $E_{\Omega} H_{*}\left(S^{2} \times \widetilde{V}_{i}\right)$ are zero. Therefore $E_{\Omega} H_{q+1}(\tilde{X}) \stackrel{\sim}{\rightarrow} E_{\Omega} H_{q+1}(M)$ is an equivariant isomorphism and the intersection signatures are equal.

Theorem 6. If $L$ is a $4 q+1$ dimensional $m$-link and $\sigma_{L}(\omega)$ is the LevineTristram signature for $\omega$ a root of unity then

$$
\sigma_{L}(\omega)=\operatorname{sig}_{L}(\omega, \omega, \omega, \ldots, \omega) .
$$

Proof. Suppose $\omega \neq 1$ is a primitive $d$ th root of unity (if $\omega=1$ then the result is trivial). According to Viro [12] $\sigma_{L}(\omega)$ may be computed in the following manner. Take a Seifert surface $U$ for $L$ (possibly connected) and push its interior into $B^{2 q+2}$ to get $U \subset B^{2 q+2}$. Form $N_{U}$ the $d$-fold branched cyclic cover of $\left(B^{2 q+2}, U\right)$ and compute $\left.\operatorname{sign}\langle\rangle\right|_{,E_{\omega}\left(H_{q+1}\left(N_{u}\right)\right)}=\sigma_{L}(\omega)$.

Let $\left(B^{2 q+2} ; V_{1}, \ldots, V_{m}\right)$ be a special $m$-tuple for $L$ and let $N_{v}$ be the $d$ fold branched cyclic cover, i.e., $N_{v}$ is associated to the map

by $\phi\left(\mu_{1}\right)=1$.

$$
\phi: H_{1}\left(B^{2 q+2}-\left(V_{1} \cup \cdots \cup V_{m}\right)\right) \rightarrow Z_{d}
$$

We show that $\left.\operatorname{sign}\langle\rangle\right|_{,E_{\omega v}\left(H_{q+1}\left(N_{\iota^{\prime}}\right)\right)}=\left.\operatorname{sign}\langle\rangle\right|_{,E_{\omega v}\left(H_{q+1}\left(N_{r}\right)\right)}$. If $\chi$ is the character on $Z_{d}, \chi(1)=\omega$ then

$$
\operatorname{sign}\left(\left.\langle,\rangle\right|_{E_{(v)}\left(H_{q+1}\left(N_{v}\right)\right)}=\left[\operatorname{Sign}\left(Z_{d}, N_{v}\right), \chi\right]\right.
$$

and

$$
\operatorname{sign}\left(\left.\langle,\rangle\right|_{E_{\omega}\left(H_{q+1}\left(N_{v}\right)\right)}\right)=\left[\operatorname{Sign}\left(Z_{d}, N\right), \chi\right] .
$$

$\partial N_{v}=\partial N_{U}$ is the branched cyclic cover of $L$ and

$$
\operatorname{Sign}\left(Z_{d}, N_{v}\right)-\operatorname{Sign}\left(Z_{d}, N_{U}\right)=\operatorname{Sign}\left(Z_{d}, N_{v} \cup\left(-N_{U}\right)\right) \text {. }
$$

We wish to see that $\operatorname{Sign}\left(Z_{d}, N_{v} \cup\left(-N_{U}\right)\right)$ is zero. As in Lemma 4, we define a map $F: \pi_{2 q+2}\left(S^{2}\right) \rightarrow R\left(Z_{d}\right)$. If $\gamma \in \pi_{2 q+2}\left(S^{2}\right)$ and $p \in S^{2}$ then represent $\gamma$ by 
a map $g$ which has a regular value at $p$. Let $N_{g}$ denote the $d$-fold branched cyclic cover of $\left(S^{2 q+2}, g^{-1}(p)\right)$. Then $F(\gamma)=\operatorname{Sign}\left(Z_{d}, N_{g}\right)$. By the argument in Lemma 4 , this map is a well-defined homomorphism. Since $\pi_{2 q+2}\left(S^{2}\right)$ is torsion, $F$ is zero. Therefore, $\operatorname{Sign}\left(Z_{d}, N_{v} \cup\left(-N_{U}\right)\right)=0$ and we may compute $\sigma_{L}(\omega)$ as $\operatorname{sign}\left(\left.\langle\rangle\right|_{,E_{\omega}\left(H_{q+1}\left(N_{v}\right)\right)}\right)$.

Let $X_{\psi}$ be the $Z_{d}$-covering space of $X$ where $\psi: H_{1}\left(B^{2 q+2}-\bigcup_{i} V_{i}\right) \rightarrow$ $Z_{d}^{m}=G$ by $\psi\left(\mu_{i}\right)=g_{i} . X_{\psi}$ is the canonical $G$-manifold over $\left(B^{2 q+2} ; V_{1}, \ldots\right.$, $\left.V_{m}\right)$ with the branched points removed. By Lemma 13,

$$
\operatorname{sign}\left(\left.\langle,\rangle\right|_{E_{\Omega}\left(H_{q+1}\left(X_{\psi}\right)\right)}\right)=\operatorname{sig}_{L}(\Omega) \quad \text { where } \Omega=(\omega, \ldots, \omega) .
$$

Let $G \stackrel{\varepsilon}{\rightarrow} Z_{d}$ be the augmentation map. Since $\varepsilon \circ \psi=\phi$ we get a hierarchy of covering spaces:

where $0 \rightarrow K \rightarrow G \stackrel{\varepsilon}{\rightarrow} Z_{d} \rightarrow 0$.

$$
G\left[\begin{array}{l}
K \quad\left[\begin{array}{l}
X_{\psi} \\
\downarrow \\
X_{\phi}
\end{array}\right. \\
Z_{d}\left[\begin{array}{l}
\downarrow \\
X
\end{array}=B^{2 q+2}-\left(\bigcup V_{i}\right)\right.
\end{array}\right.
$$

The transfer map, $H^{*}\left(X_{\phi} ; \mathbf{C}\right) \stackrel{\rho^{*}}{\rightarrow} H^{*}\left(X_{\psi} ; \mathbf{C}\right)^{K}$ is a $Z_{d}$ equivariant isomorphism. Now, $g_{1} g_{i}^{-1} \in K$ for all $i$ so if $v \in E_{\left(\omega_{1}, \ldots, \omega_{m}\right)}\left(H^{*}\left(X_{\psi}\right)\right)$ and $v$ is fixed by $K$ then $g_{1} g_{i}^{-1}(v)=\omega_{1} \omega_{i}^{-1} v$ and so $\omega_{1}=\omega_{i}$. We now have

$$
H^{*}\left(X_{\psi} ; \mathbf{C}\right)^{K}=\bigoplus_{\eta} E_{(\eta, \eta, \ldots, \eta)}\left(H^{*}\left(X_{\psi} ; \mathbf{C}\right)\right),
$$

$\eta$ runs through the $d$ th roots of unity. So $E_{\omega}\left(H^{*}\left(X_{\phi} ; \mathbf{C}\right)\right) \stackrel{\rho^{*}}{\rightarrow} E_{\Omega}\left(H^{*}\left(X_{\psi} ; \mathbf{C}\right)\right)$ is an isomorphism and the result follows since

$$
|K|\left\langle\alpha \cup \beta,\left[X_{\phi}\right]\right\rangle=\left\langle\rho^{*} \alpha \cup \rho^{*} \beta,\left[X_{\psi}\right]\right\rangle
$$

for $\alpha, \beta \in H^{q+1}\left(X_{\phi} ; \mathbf{C}\right)$.

We now suppose that each $a_{i}$ is some power of a prime $p$ and proceed to show that $\operatorname{sig}_{L}(\Omega)$ is then a cobordism invariant. We begin by showing that $\operatorname{sig}_{L}(\Omega)$ is an eigenspace signature for a cyclic $a$-fold cover $\left(a=\max a_{i}\right)$ of $B^{2 q+2}-\bigcup \nu\left(V_{i}\right)$. This cover depends upon

$$
\Omega=\left(e^{\left(2 \pi c_{1} / a_{1}\right) i}, \ldots, e^{\left(2 \pi c_{m} / a_{m}\right) i}\right)
$$

and is precisely given in the following lemma.

Lemma 7. Let $G=Z_{a_{1}} \oplus \cdots \oplus Z_{a_{m}}$, $a_{i}$ a power of a prime $p$. Let $\widehat{M}$ be the canonical $G$-manifold $M-\pi^{-1}(\nu V)$. Denote by $r$ the maximal power to occur among the $a_{i}$, i.e., $r=\max _{i} \log _{p} a_{i}$, and define $r_{j}$ by $p^{r}=a_{j} p^{r_{j}}$. Suppose $p: G \rightarrow Z_{p^{r}}$ by $p\left(\prod T_{j}^{s_{j}}\right)=T^{\sum_{j} s_{j} c_{j} p^{r_{j}}}$ where $T$ generates $Z_{p^{r}}$ and $T_{j}$ generates 
$Z_{a_{j}}$. Denote by $H$ the kernel of $p, w=e^{2 \pi i / p^{r}}$ and $w_{j}=e^{2 \pi i / a_{j}}=w^{p^{r_{j}}}$. The projection $\widehat{M} \rightarrow \widehat{M} / H$ induces an isomorphism

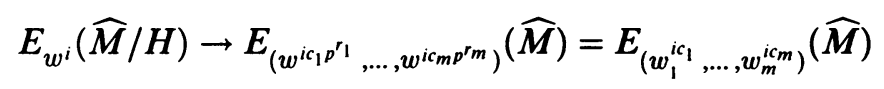

and

$$
\operatorname{sig}_{L}\left(w_{1}^{i c_{1}}, \ldots, w_{m}^{i c_{m}}\right)=\text { signature }\left.\langle,\rangle\right|_{E_{w^{i}}\left(H_{q+1}(\widehat{M} / H)\right)} .
$$

Proof. The transfer homomorphism yields an equivariant insomorphism

$$
H^{*}(\widehat{M} / H ; \mathbf{C}) \underset{\rho^{*}}{\rightarrow}\left[H^{*}(\widehat{M} ; \mathbf{C})\right]^{H}
$$

If $v \in E_{w^{i}} H^{*}(\widehat{M} / H ; \mathbf{C})$ then

$$
T_{j} \rho^{*}(v)=\rho^{*} T^{c_{j} p^{r_{j}}} v=w^{i c_{j} p^{r_{j}}} v \text { for all } j .
$$

For

$$
y \in E_{\left(w_{1}^{i c_{1}}, \ldots, w_{m}^{\left.i c_{m}\right)}\right.}(\widehat{M}),
$$

$y$ is fixed by $H$ and $y=\rho^{*} v$ for some $v$ in $H^{*}(\widehat{M} ; \mathbf{C})$. Now, $\rho^{*} T v=$ $\prod T_{j}^{b_{j}} \rho^{*} v$ where $\sum_{j} b_{j} c_{j} p^{r_{j}} \equiv 1 \bmod p^{r}$. Therefore,

$$
\rho^{*} T v=\prod_{j} w^{i c_{j} b_{j} p_{j}^{r}} \rho^{*} v=\left(w^{\sum c_{j} b_{j} p_{j}^{r_{i}}}\right) \rho^{*} v=w^{i} \rho^{*} v
$$

Theorem 8. Suppose $L^{2 q-1} \subset S^{2 q+1}$ is a slice $m$-link and that $w_{1}, \ldots, w_{m}$ are $p^{r}$ th roots of unity for $p$ a prime.

(a) If $q>1$ then $\operatorname{sig}_{L}\left(w_{1}, \ldots, w_{m}\right)=0$;

(b) If $q=1$ then $\operatorname{sig}_{L}\left(w_{1}, \ldots, w_{m}\right) \leq m-1$.

Proof. Let $\left(D^{2 q+2} ; D_{1}, \ldots, D_{m}\right)$ be a special $m$-tuple associated to $L$ which is constructed from slicing disks for $L$. By Lemmas 5 and 7 there is a $p^{r}$-fold cyclic cover $\widetilde{X}$ of $X=D^{2 q+2}-\bigcup \nu\left(D_{i}\right)$ for which

$$
\operatorname{sig}_{L}\left(w_{1}, w_{2}, \ldots, w_{m}\right)=\text { signature }\left.\langle,\rangle\right|_{E_{\eta} H_{q+1}(\widetilde{X} ; \mathbf{C})} .
$$

By Proposition 1.4 of [4],

$$
\operatorname{dim} E_{\eta} H_{q+1}(\tilde{X} ; \mathbf{C}) \leq \operatorname{dim} H_{q+1}\left(X ; Z_{p}\right)
$$

but $H_{q+1}\left(X, Z_{p}\right)=0$ when $q \neq 1$. If $q=1$ then Proposition 1.5 of [4] yields

$$
\operatorname{dim} E_{\eta} H_{1}(\tilde{X} ; \mathbf{C}) \leq \operatorname{dim} H_{1}\left(X ; Z_{p}\right)-1
$$

which is $m-1$. 


\section{BOUNDARY LINKS}

In [2], Cappell and Shaneson define a link cobordism invariant on boundry $m$-links $\left(S^{2 q+1}, L^{2 q+1}\right)$ for which $m \leq q-1$. They compute boundary link cobordism in terms of the homology surgery obstruction $\Gamma$-groups. Boundary link cobordism is

$$
\frac{\Gamma_{2 q+2}\left(Z\left[F^{m}\right] \rightarrow Z\right)}{\operatorname{Im}\left(L_{2 q+2}\left(Z\left[F^{m}\right]\right)\right)} \oplus m P_{n+1}
$$

in the notation of [2]. Here $F^{m}$ is the free group on $m$ letters. The cobordism invariant is defined via the map

$$
\Gamma_{2 q+2}\left(Z\left[F^{m}\right] \rightarrow Z\right) \rightarrow \frac{\Gamma_{2 q+2}\left(Z\left[Z^{m}\right] \rightarrow Z\right)}{\operatorname{Im}\left(L_{2 q+2}\left(Z\left[Z^{m}\right]\right)\right)} .
$$

The elements of the $\Gamma$-group are 3-tuples with one entry being the $Z\left[F^{m}\right]$ intersection matrix of an $F^{m}$-covering space associated to $L$. This cover is an $F^{m}$-cover of a particular special $m$-tuple associated to $L$. One constructs the $m$-tuple by pushing the interior of a Seifert surface to $L$ into the interior of $B^{2 q+2}$. Since every boundary link is boundary cobordant to a simple link, we can assume that $L$ is a simple boundary link. Let $M\left(t_{1}, \ldots, t_{m}\right)$ be the image in $Z\left[Z^{m}\right]$ of the intersection matrix. Cappell and Shaneson detect an infinite family of 2-links which are not sliced but whose knot components are sliced by the map $L \rightarrow$ signature $M(\omega, \omega, \ldots, \omega)$ for $\omega$ a prime root of unity and $q$ odd or $L \rightarrow$ signature $i M(\omega, \omega, \ldots, \omega)$ if $q$ is even. Under the assumption that the Seifert surfaces are highly connected, the intersection from on a $G$ cover is given by the image of

$$
H_{q+1}\left(B-\bigcup V_{i}\right) \rightarrow H_{q+1}\left(B-\bigcup V_{i}\right) \otimes_{Z[F]} Z[G] .
$$

The invariant $\operatorname{sig}_{L}\left(\omega_{1}, \ldots, \omega_{m}\right)$ is then the signature of $M\left(\omega_{1}, \ldots, \omega_{m}\right)$ or $i M\left(\omega_{1}, \ldots, \omega_{m}\right)$. We now see that signature $(\boldsymbol{M}(w, w, \ldots, w))$ is the LevineTristram signature.

On the restricted class of boundary links, the invariant $\operatorname{sig}_{L}$ may be interpreted via a map

$$
s: \frac{\Gamma_{2 q+2}\left(Z\left[Z^{m}\right] \rightarrow Z\right)}{L_{2 q+2} Z\left[Z^{m}\right]} \rightarrow \operatorname{Fun}\left(T^{m}, Z\right) .
$$

If $N\left(t_{1}, \ldots, t_{m}\right)$ arises from $L\left(Z\left[Z^{m}\right]\right)$ then $f_{N}: T^{m} \rightarrow Z$ given by $f_{N}(\Omega)=$ signature $N(\Omega)$ is a constant function since $f_{N}$ is continuous on

$$
\left\{\Omega \in T^{m} / \operatorname{det} N(\Omega) \neq 0\right\},
$$

i.e.,

$$
\text { signature } N(\Omega)=\text { signature } N(1,1, \ldots, 1) .
$$

If $N$ is a form from $\Gamma_{2 q+2}\left(Z\left[Z^{m}\right] \rightarrow Z\right)$ then $s$ is defined by $s(N)(\Omega)=$ signature $(N(\Omega) \oplus-N(1,1, \ldots, 1))$. By the above remark $s$ is well defined. 
Also note that $s(N): T^{m} \rightarrow Z$ is continuous (and therefore constant) on the components of $T^{m}-\left\{\Omega \in T^{m} / \operatorname{det} N(\Omega)=0\right\}$.

We now give a formula for $\operatorname{sig}_{L}$ when $L$ is a boundary link. If $V_{1} \cup \cdots \cup V_{m}$ is a boundary type Seifert surface for $L$, i.e., $\partial V_{i}=L_{i}$, then $\operatorname{sig}_{L}$ may be computed in terms of the Seifert matrix. Let $S=\left[A_{i j}\right]$ be the Seifert matrix for $U V_{i} . S$ is a matrix of matrices with $A_{i i}$ being the $l_{i} \times l_{i}$ matrix of $V_{i}$ (see [5] for details). One can form the special $m$-tuple by pushing the interior of $V_{i}$ into the interior of $B^{2 q+2}$ along isotopies arriving at $\widehat{V}_{i}$. The usual $G$ cover of $B^{2 q+2}-\bigcup \widehat{V}_{i}$ can be decomposed into a union of balls: each ball is $B^{2 q+2}$-(path of the isotopies). The intersection pairing can then be computed. This well-known Mayer-Vietoris argument combined with some matrix algebra establishes the following formula.

Theorem 9. Suppose $L$ is a $2 q-1$ dimensional boundary m-link with Seifert matrix $S$ as above. Then

$$
\operatorname{sig}_{L}\left(w_{1}, \ldots, w_{m}\right)= \begin{cases}\operatorname{signature}\left(i(I-W)\left(-S W^{-1}-S^{T}\right)\right. & \text { if } q \text { is even } \\ \operatorname{signature}\left((I-W)\left(-S W^{-1}+S^{T}\right)\right) & \text { if } q \text { is odd }\end{cases}
$$

where

$$
W=\left(\begin{array}{cccc}
{\left[w_{1}\right]} & & & \\
& {\left[w_{2}\right]} & & 0 \\
0 & & \ddots & \\
& & & {\left[w_{m}\right]}
\end{array}\right), \quad\left[w_{i}\right]=w_{i} I_{l_{1} \times l_{i}},
$$

and $w_{i}$ is an $a_{i}$ th root of unity.

Examples. (1) Let $K$ be a knot with $\sigma_{K}(w) \neq 0$. Form $L$ a two component parallel link by taking a parallel push off $K^{\prime}$ of $K$ so $L=K \cup K^{\prime}$. The link $L$ is weakly sliced since $L$ bounds a cylinder in $S^{n+1}$ and $\sigma_{L}\left(S^{1}\right)=0$. This example was used in [10]. It demonstrates that $\operatorname{sig}_{L}$ is not a weak cobordism invariant but it relies upon the Levine-Tristram signature of a component.

(2) These examples consist of two component links for which the LevineTristram signature of the link and each component is zero but $\operatorname{sig}_{L}$ is nonzero. Example $(2 \mathrm{a})$ is a $4 k+1$ dimensional link and example (2b) is a $4 k-1$ dimensional link.

(a) Consider $\mathscr{F}=\left(Z\left[F^{2}\right] \oplus Z\left[F^{2}\right], \phi, \mu\right)$ where $F^{2}=$ free group $\langle s, t\rangle, \phi$ is the bilinear form,

$$
\phi(s, t)=\left[\begin{array}{cc}
t^{-1} s\left(t-t^{-1}\right)-\left(t-t^{-1}\right) s^{-1} t & 1 \\
1 & t^{-1} s\left(s-s^{-1}\right)-\left(s-s^{-1}\right) s^{-1} t
\end{array}\right]
$$

and $\mu(a, b)=a t^{-1} s\left(t-t^{-1}\right) \bar{a}+a \bar{b}+b t^{-1} s\left(s-s^{-1}\right) \bar{b}$. The triple $\mathscr{F}$ is in $\Gamma_{4 k}\left(Z\left[F^{2}\right] \stackrel{\varepsilon}{\rightarrow} Z\right)$ where $\varepsilon$ is the augmentation. According to Cappell and Shaneson, $\mathscr{F}$ can be associated to a boundary link $L$. The Levine-Tristram signatures of the components arise from $\operatorname{sign} \phi(1, \eta)$ and $\operatorname{sign} \phi(\eta, 1)$ for $\eta$ a 
root of unity. However,

$$
\phi(1, \eta)=\left[\begin{array}{cc}
-\left(\eta-\eta^{-1}\right)^{3} & 1 \\
1 & 0
\end{array}\right] \text { and } \phi(\eta, 1)=\left[\begin{array}{cc}
0 & 1 \\
1 & \left(\eta-\eta^{-1}\right)^{3}
\end{array}\right]
$$

Similarly the Levine-Tristram signature of $L$ is $\operatorname{sign} \phi(\eta, \eta)$ but

$$
\phi(\eta, \eta)=\left[\begin{array}{ll}
0 & 1 \\
1 & 0
\end{array}\right]
$$

Let $\omega=e^{2 \pi i / 6}=1 / 2+(\sqrt{3} / 2) i$. The mixed invariant $\operatorname{sign} \phi\left(\omega, \omega^{2}\right)=\operatorname{sign}\left[\begin{array}{ll}3 & 1 \\ 1 & 3\end{array}\right]$ $=2$ so $\operatorname{sig}_{L}\left(\omega, \omega^{2}\right)=2$.

(b) Consider $\mathscr{F}=\left(Z\left[F^{2}\right] \otimes Z\left[F^{2}\right], \phi, \mu\right)$ where

$$
\phi(s, t)=\left[\begin{array}{cc}
t^{-1} s\left(t-t^{-1}\right)^{2}-\left(t-t^{-1}\right)^{2} s^{-1} t & -1 \\
1 & t^{-1} s\left(s-s^{-1}\right)^{2}-\left(s-s^{-1}\right)^{2} s^{-1} t
\end{array}\right]
$$

and $\mu(a, b)=a t^{-1} s\left(t-t^{-1}\right)^{2} \bar{a}-a \bar{b}+b t^{-1} s\left(s-s^{-1}\right)^{2} \bar{b}$. The triple $\mathscr{F}$ is in $\Gamma_{4 k+2}\left(Z\left[F^{2}\right] \stackrel{\varepsilon}{\rightarrow} Z\right)$ and is associated to boundary links, say one is $L$. One can calculate the signatures as in (a) with the results: $\operatorname{sig}_{L}(\eta, 1)=\operatorname{sig}_{L}(1, \eta)=$ $\operatorname{sig}_{L}(\eta, \eta)=0$ are the Levine-Tristram signatures and $\operatorname{sig}_{L}\left(\omega, \omega^{2}\right)=2$ for $\omega=e^{2 \pi i / 6}$.

\section{THE ClASSICAL DIMENSION}

In this section we depart from our previous approach and consider only branched covers of $B^{4}$ instead of covers of surface complements. From our perspective the case $n=1$ differs from the high dimensional case since the surfaces inside $B^{4}$ must intersect if the pairwise linking numbers are nonzero. These intersections change the argument only slightly. The homology $H_{1}\left(B^{4}-\bigcup V_{i}\right)$ is still $Z^{m}$. An intersection point of $V_{i}$ and $V_{j}$ has a neighborhood of the form $\left(D^{2} \times D^{2} ; D^{2} \times 0 \cup 0 \times D^{2}\right)$. The cover of the complement $\left(D^{2}-0\right) \times\left(D^{2}-0\right) \rightarrow\left(D^{2}-0\right) \times\left(D^{2}-0\right)$ given by $\left(r_{1} e^{i \theta}, r_{2} e^{i \psi}\right) \mapsto\left(r_{1} e^{i a_{1} \theta}, r_{2} e^{i a_{2} \psi}\right)$ can be completed to $D^{2} \times D^{2}$ by the same formula. We can then complete the cover of $B^{4}-\bigcup V_{i}$ to a branched cover $B_{V} \rightarrow B^{4}$. The fixed point set of $g_{i}^{a}$ is $V_{i}$ and the fixed point set of $g_{i}^{a} g_{j}^{b}$ is the finite set of points $V_{i} \cap V_{j}$. Other group elements formed from the product of more than three generators do not have fixed points. In order to see that $\operatorname{Sign}\left(G, B_{V}\right)$ is an invariant we choose another set of surfaces $V^{\prime}$, construct the branched cover $B_{V^{\prime}}$ and observe that $\left.\operatorname{Sign}\left(G, B_{V} \cup-B_{V^{\prime}}\right)\right)=0$. The $G$-signature is zero by the $G$-signature theorem; the contribution from the isolated fixed points is given by

$$
\begin{aligned}
& \operatorname{Sign}\left(g_{i}^{r} g_{j}^{s}, B_{V} \cup\left(-B_{V^{\prime}}\right)\right) \\
& \quad=|G| \cdot \frac{1}{a_{i} a_{j}}\left(\left|V_{i} \cap V_{j}\right|-\left|V_{i}^{\prime} \cap V_{j}^{\prime}\right|\right) \cot \left(\frac{\pi r}{a_{i}}\right) \cdot \cot \left(\frac{\pi s}{a_{j}}\right) .
\end{aligned}
$$

Since $\left|V_{i} \cap V_{j}\right|=\left|V_{i}^{\prime} \cap V_{j}^{\prime}\right|=\operatorname{lk}\left(L_{i}, L_{j}\right)$ with multiplicities, the contribution is zero. 
Versions of this classical link invariant were defined by Cooper [3] and, in essence, by Gilmer [4]. The author is grateful to P. Gilmer for alerting him to these works. Cooper constructs Seifert surfaces to the link components with special types of intersections, associates a Seifert type of pairing and proceeds along lines similar to Tristram. Gilmer computes Casson-Gordon invariants of 3-manifolds described by surgery along $L$. One performs this calculation by replacing certain link components by cable knots. The Tristram signature of this altered link is the invariant (see Theorem 3.6 of [4]).

\section{REFERENCES}

1. M. F. Atiyah and I. M. Singer, The index of elliptic operators. III, Ann. of Math. 87 (1968), 546-604.

2. S. Cappell and J. L. Shaneson, Link cobordism, Comment. Math. Helv. 55 (1980), 20-49.

3. D. Cooper, The universal abelian cover of a link, Low-Dimensional Topology ( $R$. Brown and T. L. Thickstun, eds.), Cambridge Univ. Press, 1985.

4. P. Gilmer, Configurations of surfaces in 4-manifolds, Trans. Amer. Math. Soc. 264 (1981), 353-380.

5. Ki Hyoung Ko, Seifert matrices and boundary link cobordisms, Trans. Amer. Math. Soc. 299 (1987), 657-681.

6. J. Levine, Knot cobordism groups in codimension two, Comment. Math. Helv. 44 (1969), 229-244.

7. _ Invariants of knot cobordism, Invent. Math. 8 (1969), 98-110.

8. J. Milnor, Infinite cyclic covers, Conference on the Topology of Manifolds (J. G. Hocking, ed.), Prindle, Weber and Schmidt, 1968.

9. L. Smolinsky, Doubly sliced knots which are not the double of a disk, Trans. Amer. Math. Soc. 298 (1986), 723-732.

10. _ Casson-Gordon invariants of some 3-fold branched covers of knots, Topology Appl. (to appear).

11. A. G. Tristram, Some cobordism invariants for links, Proc. Cambridge Philos. Soc. 66 (1969), 251-264.

12. O. J. Viro, Branched coverings of manifolds with boundary and link invariants. I, Math. USSR Izv. 7 (1973), 1239-1256.

13. G. W. Whitehead, Elements of homotopy theory, Graduate Texts in Math., vol. 61, SpringerVerlag, New York, 1978.

Department of Mathematics, Yale University, New Haven, Connecticut 06520

Current address: Department of Mathematics, Louisiana State University, Baton Rouge, Louisiana 70803 